\title{
MODULAR AND LOWER-MODULAR ELEMENTS OF LATTICES OF SEMIGROUP VARIETIES
}

\author{
V. YU. SHAPRYNSKIǏ \\ COMMUNICATED BY L. N. SHEVRIN
}

\begin{abstract}
The paper contains three main results. First, we show that if a commutative semigroup variety is a modular element of the lattice Com of all commutative semigroup varieties then it is either the variety $\mathcal{C O M}$ of all commutative semigroups or a nil-variety or the join of a nil-variety with the variety of semilattices. Second, we prove that if a commutative nil-variety is a modular element of Com then it may be given within $\mathcal{C O} \mathcal{M}$ by 0 -reduced and substitutive identities only. Third, we completely classify all lower-modular elements of Com. As a corollary, we prove that an element of Com is modular whenever it is lower-modular. All these results are precise analogues of results concerning modular and lower-modular elements of the lattice of all semigroup varieties obtained earlier by Jezek, McKenzie, Vernikov, and the author. As an application of a technique developed in this paper, we provide new proofs of the 'prototypes' of the first and the third our results.
\end{abstract}

\section{INTRODUCTION AND SUMMARY}

There are several papers where special elements of the lattice of all semigroup varieties were examined. Results in this area obtained before 2009 have been overviewed in Section 14 of the survey [11]. Recall the definitions of special elements mentioned in the present paper. An element $x$ of a lattice $\langle L ; \vee, \wedge\rangle$ is called

$$
\begin{array}{lll}
\text { modular if } & \forall y, z \in L: & y \leq z \longrightarrow(x \vee y) \wedge z=(x \wedge z) \vee y, \\
\text { lower-modular if } & \forall y, z \in L: & x \leq y \longrightarrow x \vee(y \wedge z)=y \wedge(x \vee z), \\
\text { distributive if } & \forall y, z \in L: & x \vee(y \wedge z)=(x \vee y) \wedge(x \vee z),
\end{array}
$$

and neutral if, for all $y, z \in L$, the sublattice generated by the elements $x, y$, and $z$ is distributive. Upper-modular and codistributive elements are defined dually to lower-modular and distributive ones respectively. It is evident that

2000 Mathematics Subject Classification. Primary 20M07, secondary 08B15.

Key words and phrases. Semigroup, variety, lattice of varieties, commutative variety, modular element, lower-modular element.

The work was partially supported by the Russian Foundation for Basic Research (grants No. 09-01-12142, 10-01-00524) and the Federal Education Agency of the Russian Federation (project No. 2.1.1/3537). 
every [co]distributive element is lower-modular [upper-modular] and that every neutral element is distributive, codistributive, and modular.

The lattice of all semigroup varieties is denoted by SEM. Neutral elements of this lattice were determined by Volkov in [18]. A description of distributive and lower-modular elements in SEM was obtained by Vernikov and the author in [15] and [10] respectively. An essential information about modular elements of SEM was found in [5, 12]. Some of the mentioned results are interesting from the point of view of this paper. To formulate these results, we need some definitions and notation.

For convenience, we call a semigroup variety modular if it is a modular element of the lattice SEM, and adopt analogous agreement for all other types of special elements. As usual, we replace a pair of identities $w x=$ $x w=w$ where the letter $x$ does not occur in the word $w$ by the symbolic identity $w=0$. Identities of the form $w=0$ as well as varieties given by such identities are called 0-reduced. An identity $u=v$ is called substitutive if the words $u$ and $v$ depend on the same letters and the word $v$ may be obtained from $u$ by renaming of letters. Recall that a semigroup variety is called a nil-variety if it consists of nil-semigroups (or, equivalently, if it satisfies an identity of the form $x^{n}=0$ for some $n$ ). It is evident that every 0 -reduced variety is a nil-variety. By $\mathcal{T}, \mathcal{S} \mathcal{L}$, and $\mathcal{S E M}$ we denote the trivial variety, the variety of all semilattices, and the variety of all semigroups respectively.

Proposition 1.1. If a semigroup variety $\mathcal{V}$ is modular then either $\mathcal{V}=\mathcal{S E M}$ or $\mathcal{V}=\mathcal{M} \vee \mathcal{N}$ where $\mathcal{M}$ is one of the varieties $\mathcal{T}$ or $\mathcal{S} \mathcal{L}$, while $\mathcal{N}$ is a nilvariety.

This fact was proved (in slightly weaker form and some other terminology) in [5], Proposition 1.6; a deduction of Proposition 1.1 from [5, Proposition 1.6] is given explicitly in [12, Proposition 2.1]. Proposition 1.1, together with Lemma 2.1 formulated below, completely reduces the problem of description of modular varieties to the nil-case. The following necessary condition for a nil-variety to be modular (stronger than one given by Proposition 1.1) is true.

Proposition 1.2 ([12, Theorem 2.5]). If a nil-variety of semigroups is modular then it may be given by 0-reduced and substitutive identities only.

The last earlier result we cite here is the following

Proposition 1.3 ([10, Theorem 1.1]). A semigroup variety $\mathcal{V}$ is lowermodular if and only if either $\mathcal{V}=\mathcal{S E \mathcal { M }}$ or $\mathcal{V}=\mathcal{M} \vee \mathcal{N}$ where $\mathcal{M}$ is one of the varieties $\mathcal{T}$ or $\mathcal{S} \mathcal{L}$, while $\mathcal{N}$ is a 0 -reduced variety.

It is natural to study special elements not only in the whole lattice SEM but also in its most important sublattices. One of such sublattices is the lattice Com of all commutative semigroup varieties. This lattice is intensively studied and several deep results were obtained here. The lattice Com contains an isomorphic copy of any finite lattice (this follows from results of the 
papers [1] and [8]), so it does not satisfy any non-trivial lattice identity. On the other hand, this lattice is countably infinite [7]. Some parametrization of the lattice Com was suggested in [6]. The study of special elements in Com was started by the author in [9] where distributive and neutral elements in this lattice were described.

Main results of this paper are precise analogoues of Propositions 1.1-1.3 for the lattice Com. To formulate these results, we need some additional definitions and notation. We call a commutative semigroup variety modular in Com if it is a modular element of the lattice Com, and adopt analogous agreement for all other types of special elements. By $\mathcal{C O} \mathcal{M}$ we denote the variety of all commutative semigroups. A commutative semigroup variety is called 0-reduced in $\mathbf{C o m}$ if it is defined within $\mathcal{C O M}$ by 0-reduced identities only. Our main results are the following three theorems.

Theorem 1.4. If a commutative semigroup variety $\mathcal{V}$ is modular in Com then either $\mathcal{V}=\mathcal{C O} \mathcal{M}$ or $\mathcal{V}=\mathcal{M} \vee \mathcal{N}$ where $\mathcal{M}$ is one of the varieties $\mathcal{T}$ or $\mathcal{S} \mathcal{L}$, while $\mathcal{N}$ is a nil-variety.

Theorem 1.5. If a commutative nil-variety of semigroups is modular in Com then it may be given within the variety $\mathcal{C O M}$ by 0 -reduced and substitutive identities only.

Theorem 1.6. A commutative semigroup variety $\mathcal{V}$ is lower-modular in Com if and only if either $\mathcal{V}=\mathcal{C O} \mathcal{M}$ or $\mathcal{V}=\mathcal{M} \vee \mathcal{N}$ where $\mathcal{M}$ is one of the varieties $\mathcal{T}$ or $\mathcal{S} \mathcal{L}$, while $\mathcal{N}$ is a 0 -reduced in $\mathbf{C o m}$ variety.

The technique developed in the course of proving these theorems permits to give new proofs of Propositions 1.1 and 1.3. Moreover, in fact Propositions 1.1 and 1.3 may be verified by practically the same arguments as Theorems 1.4 and 1.6 respectively. It seems for us that these new proofs of earlier results are of some independent interest, and we include these proofs in the paper.

The article consists of four sections. Section 2 contains some auxuliary results. In Section 3 we prove Theorems 1.4-1.6 and provide new proofs of Propositions 1.1 and 1.3. We deduce all these results from some general assertion about modular and lower-modular elements of the subvariety lattice of an arbitrary overcommutative variety (see Proposition 3.3). Finally, in Section 4 we provide some corollaries of Theorem 1.6.

\section{Preliminaries}

We start with some definitions and auxiliary results. Suppose that $I$ is a lattice identity of the form

$$
s\left(x_{0}, x_{1}, \ldots, x_{n}\right)=t\left(x_{0}, x_{1}, \ldots, x_{n}\right) .
$$

We say that an element $x$ of a lattice $L$ is an I-element of this lattice if

$$
\forall x_{1}, \ldots, x_{n} \in L: \quad s\left(x, x_{1}, \ldots, x_{n}\right)=t\left(x, x_{1}, \ldots, x_{n}\right) .
$$


Note that all types of elements mentioned above are partial cases of $I$ elements. For distributive and codistributive elements this is evident. It is well-known that an element $x \in L$ is neutral if and only if

$$
\forall y, z \in L: \quad(x \vee y) \wedge(y \vee z) \wedge(z \vee x)=(x \wedge y) \vee(y \wedge z) \vee(z \wedge x)
$$

(see Theorem III.2.4. in [3], for instance). Finally, modular elements can be defined by the condition

$$
\forall y, z \in L: \quad(x \vee y) \wedge(y \vee z)=(x \wedge(y \vee z)) \vee y
$$

lower-modular ones by the condition

$$
\forall y, z \in L: \quad(x \vee y) \wedge(x \vee z)=((x \vee y) \wedge z) \vee x
$$

and upper-modular ones by the condition dual to the latter one.

The following lemma can be obtained by a combination of Corollary 2.1 and Lemma 2.4 of [9].

Lemma 2.1. Let I be a lattice identity satisfied by distributive lattices. $A$ [commutative] semigroup variety $\mathcal{V}$ is an I-element of the lattice SEM $[$ respectively $\mathbf{C o m}]$ if and only if the variety $\mathcal{V} \vee \mathcal{S L}$ has this property.

Lemma 2.2. Every 0 -reduced [in $\mathbf{C o m}$ ] semigroup variety is modular [in Com].

The fact that a 0 -reduced variety is modular was noted for the first time in [16], Corollary 3, and rediscovered in some other terms in [5], Proposition 1.1. The 'commutative part' of Lemma 2.2 was verified in [9], Proposition 2.1. Note that, in fact, Lemma 2.2 readily follows from [4], Proposition 2.2 .

Lemma 2.3. A [commutative] nil-variety of semigroups is lower-modular [in $\mathbf{C o m}]$ if and only if it is 0-reduced [in Com].

For lower-modular varieties, this fact was proved in [16], Corollary 3 (the 'if' part) and [13], Corollary 2.7 (the 'only if' part); for lower-modular in Com varieties it was verified in [9], Proposition 2.2.

Lemma 2.4 ([9, Proposition 2.3]). A 0-reduced in Com semigroup variety is upper-modular in $\mathbf{C o m}$ if and only if it satisfies the identity $x^{2} y=0$.

If $u$ is a word and $x$ is a letter then we denote by $c(u)$ the content of $u$, that is, the set of all letters occurring in $u$; further, $h(u)$ [respectively $t(u)$ ] denotes the first [the last] letter of $u$, and $\ell_{x}(u)$ is the number of occurences of $x$ in $u$. The symbol $\equiv$ stands for the equality relation on the free semigroup of a countably infinite rank. We denote by var $\Sigma$ the semigroup variety given by the identity system $\Sigma$. Put

$$
\begin{aligned}
& \mathcal{L Z}=\operatorname{var}\{x y=x\}, \\
& \mathcal{R Z}=\operatorname{var}\{x y=y\}, \\
& \mathcal{P}=\operatorname{var}\left\{x y=x^{2} y, x^{2} y^{2}=y^{2} x^{2}\right\}, \\
& \overleftarrow{\mathcal{P}}=\operatorname{var}\left\{x y=x y^{2}, x^{2} y^{2}=y^{2} x^{2}\right\}
\end{aligned}
$$


The first statement of the following lemma is evident, while the second one is verified in [2], Lemma 7.

Lemma 2.5. The identity $u=v$ holds:

(i) in the variety $\mathcal{L} \mathcal{Z}$ if and only if $h(u) \equiv h(v)$;

(ii) in the variety $\mathcal{P}$ if and only if $c(u)=c(v)$ and either $\ell_{t(u)}(u)>1$ and $\ell_{t(v)}(v)>1$ or $\ell_{t(u)}(u)=\ell_{t(v)}(v)=1$ and $t(u) \equiv t(v)$.

A semigroup variety is called periodic if it consists of periodic semigroups. Lemma 2 of the paper [17] and the proof of Proposition 1 of the same paper imply the following

Lemma 2.6. If a periodic semigroup variety $\mathcal{V}$ does not contain the varieties $\mathcal{L} \mathcal{Z}, \mathcal{R} \mathcal{Z}, \mathcal{P}$, and $\overleftarrow{\mathcal{P}}$ then $\mathcal{V}=\mathcal{M} \vee \mathcal{N}$ where the variety $\mathcal{M}$ is generated by a monoid, while $\mathcal{N}$ is a nil-variety.

Let $\mathcal{V}$ be a commutative nil-variety of semigroups. We denote by $\operatorname{ZR}(\mathcal{V})$ the variety given by the commutative law and all 0-reduced identities that hold in $\mathcal{V}$. It is clear that $\mathrm{ZR}(\mathcal{V})$ is the least 0-reduced in Com variety that contains $\mathcal{V}$. For any natural $n$ we denote by $\mathcal{A}_{n}$ the variety of periodic Abelian groups whose exponent divides $n$.

Lemma 2.7 ([9, Lemma 2.5]). If a commutative semigroup variety $\mathcal{V}$ satisfies the identity $x^{n}=0$ then $\mathcal{V} \vee \mathcal{A}_{n}=\operatorname{ZR}(\mathcal{V}) \vee \mathcal{A}_{n}$.

We need the following two well known and easily verified technical remarks about identities of nilsemigroups.

Lemma 2.8. Let $\mathcal{V}$ be a nil-variety of semigroups.

(i) If the variety $\mathcal{V}$ satisfies an identity $u=v$ with $c(u) \neq c(v)$ then $\mathcal{V}$ satisfies also the identity $u=0$.

(ii) If the variety $\mathcal{V}$ satisfies an identity of the form $u=$ vuw where at least one the words $v, w$ is non-empty then $\mathcal{V}$ satisfies also the identity $u=0$.

A semigroup variety $\mathcal{V}$ is called overcommutative if $\mathcal{V} \supseteq \mathcal{C O} \mathcal{M}$. An identity $u=v$ is called balanced if $\ell_{x}(u)=\ell_{x}(v)$ for every letter $x$. For convenience of references, we formulate the following two generally known facts.

Lemma 2.9. An arbitrary semigroup variety is either periodic or overcommutative.

Lemma 2.10. If an overcommutative semigroup variety satisfies some identity then this identity is balanced.

\section{PRoOfS OF MAIN RESUlts}

We need some new definitions and notation. We denote by $\mathrm{F}$ the free semigroup over a countably infinite alphabet. If $u \in \mathrm{F}$ then we denote by $\ell(u)$ the length of $u$. For an arbitrary semigroup variety $\mathcal{X}$, we denote by 
$\mathrm{L}(\mathcal{X})$ its subvariety lattice, by $\mathrm{F}(\mathcal{X})$ its free semigroup over a countably infinite alphabet, by $\mathrm{F}_{c}(\mathcal{X})$ its free semigroup over an alphabet $c$, and by $\mathrm{L}(\mathrm{F}(\mathcal{X}))$ the lattice of fully invariant congruences on $\mathrm{F}(\mathcal{X})$. The equality relation on $\mathrm{F}(\mathcal{X})$ and $\mathrm{F}_{c}(\mathcal{X})$ will be denoted by $\equiv$. Let now $\mathcal{X}$ be an overcommutative variety and $U \in \mathrm{F}(\mathcal{X})$. Lemma 2.10 permits to define the length of $U$ (denoted by $\ell(U)$ ) as the length of an arbitrary word $u \in U$, the content of $U$ (denoted by $c(U)$ ) as the content of an arbitrary word $u \in U$, and the number of occurerences of a letter $x$ in $U$ (denoted by $\ell_{x}(U)$ ) as the number of occurences of $x$ in an arbitrary word $u \in U$. The $\mathcal{X}$-image of an arbitrary word is its image under the natural homomorphism from $\mathrm{F}$ to $\mathrm{F}(\mathcal{X})$. We call elements $U$ and $V$ of $\mathrm{F}(\mathcal{X})$ equivalent if $U \equiv \xi(V)$ for some automorphism $\xi$ on $\mathrm{F}(\mathcal{X})$ (in other words, if $|c(U)|=|c(V)|$ and $U \equiv \xi(V)$ for some isomorphism $\xi$ from $\mathrm{F}_{c(V)}(\mathcal{X})$ to $\left.\mathrm{F}_{c(U)}(\mathcal{X})\right)$. We call two words $\mathcal{X}$-equivalent if their $\mathcal{X}$-images are equivalent. We say that an element $W \in \mathrm{F}(\mathcal{X})$ is $\mathcal{X}$-unstable if $\xi(W) \not \equiv W$ for any non-trivial automorphism $\xi$ on $\mathrm{F}_{c(W)}(\mathcal{X})$. Otherwise the element $W$ is called $\mathcal{X}$-stable. A word is called $\mathcal{X}$-[un] stable if its $\mathcal{X}$-image is [un]stable. The group of automorphisms [the semigroup of endomorphisms] of a semigroup $S$ is denoted by $\operatorname{Aut}(S)[$ respectively $\operatorname{End}(S)]$.

The following lemma plays the key role in what follows.

Lemma 3.1. Let $\mathcal{X}$ be an overcommutative semigroup variety and let $u, v, s$, and $t$ be $\mathcal{X}$-unstable and pairwise $\mathcal{X}$-non-equivalent words with the same length and the same content. If a semigroup variety $\mathcal{V}$ is either a modular or a lower-modular element of the lattice $\mathrm{L}(\mathcal{X})$ and $\mathcal{V}$ satisfies the identities $u=v$ and $s=t$ then $\mathcal{V}$ satisfies also the identity $u=s$.

Proof. Let $\ell$ be the length of the words $u, v, s$, and $t$, and let $c$ be the content of these words. Denote by $U, V, S$, and $T$ the $\mathcal{X}$-images of the words $u, v, s$, and $t$ respectively. Let $\alpha$ be the fully invariant congruence on $\mathrm{F}(\mathcal{X})$ corresponding to the variety $\mathcal{V}$. Then $U \alpha V$ and $S \alpha T$. We aim to prove that $U \alpha S$.

Consider any two distinct elements $A, B \in\{U, V, S, T\}$ and any two automorphisms $\xi$ and $\zeta$ on $\mathrm{F}(\mathcal{X})$. We shall prove that the pairs $\{\xi(A), \xi(B)\}$ and $\{\zeta(A), \zeta(B)\}$ either coincide or do not intersect. Suppose that

$$
\{\xi(A), \xi(B)\} \cap\{\zeta(A), \zeta(B)\} \neq \varnothing .
$$

Acting by the automorphism $\xi^{-1}$ we get $\{A, B\} \cap\{\varphi(A), \varphi(B)\} \neq \varnothing$ where $\varphi=\xi^{-1} \zeta$. Since the elements $A$ and $B$ are not equivalent, $A \not \equiv \varphi(B)$ and $B \not \equiv \varphi(A)$, so either $A \equiv \varphi(A)$ or $B \equiv \varphi(B)$. We may suppose without loss of generality that $A \equiv \varphi(A)$. Since $\varphi$ is an automorphism, the restriction $\bar{\varphi}$ of $\varphi$ on $\mathrm{F}_{c(A)}(\mathcal{X})$ is an isomorphism from $\mathrm{F}_{c(A)}(\mathcal{X})$ to $\mathrm{F}_{\varphi(c(A))}(\mathcal{X})$. But $\varphi(c(A))=c(\varphi(A))=c(A)$, so $\bar{\varphi}$ is an automorphism on $\mathrm{F}_{c(A)}(\mathcal{X})$. Since $A$ is unstable, the automorphism $\bar{\varphi}$ is trivial. Further, $c(A)=c(B)$, whence $\varphi(B) \equiv \bar{\varphi}(B) \equiv B$. Acting by the automorphism $\xi$ on the equality $\varphi(B) \equiv$ $B$, we get $\xi(B) \equiv \zeta(B)$, whence $\{\xi(A), \xi(B)\}=\{\zeta(A), \zeta(B)\}$. 
Further, consider the set

$$
\mathfrak{W}=\{W \in \mathrm{F}(\mathcal{X}) \mid \text { either } \ell(W)>\ell \text { or } \ell(W)=\ell \text { and }|c(W)|<|c|\} .
$$

Since an arbitrary automorphism $\xi$ on $\mathrm{F}(\mathcal{X})$ does not change the parameters $\ell(W)$ and $|c(W)|$ of an element $W \in \mathrm{F}(\mathcal{X})$, none of the elements $\xi(U), \xi(V), \xi(S)$, and $\xi(T)$ belongs to $\mathfrak{W}$. Therefore none of the pairs $\{\xi(U), \xi(S)\}$ and $\{\xi(V), \xi(T)\}$ intersects with $\mathfrak{W}$. Further, let $\xi, \zeta \in \operatorname{Aut}(\mathrm{F}(\mathcal{X}))$. Then $\{U, S\} \cap\left\{\xi^{-1} \zeta(V), \xi^{-1} \zeta(T)\right\}=\varnothing$ because the elements $U, V, S$, and $T$ are pairwise non-equivalent. Therefore $\{\xi(U), \xi(S)\} \cap$ $\{\zeta(V), \zeta(T)\}=\varnothing$. Combining these observations with the arguments from the previous paragraph, we obtain that there is a partition of the set $\mathrm{F}(\mathcal{X})$ whose non-singleton classes are $\mathfrak{W}$ and 2-element sets of the form $\{\xi(U), \xi(S)\}$ and $\{\xi(V), \xi(T)\}$ where $\xi$ runs over $\operatorname{Aut}(\mathrm{F}(\mathcal{X}))$. Denote the equivalence relation corresponding to this partition by $\gamma$. By the same arguments, there are partitions of $\mathrm{F}(\mathcal{X})$ whose non-singleton classes are:

- $\mathfrak{W}$ and pairs $\{\xi(V), \xi(T)\}$ where $\xi$ runs over $\operatorname{Aut}(\mathrm{F}(\mathcal{X}))$;

- $\mathfrak{W}$ and pairs $\{\xi(U), \xi(V)\}$ where $\xi$ runs over $\operatorname{Aut}(\mathrm{F}(\mathcal{X}))$.

Denote the equivalence relation corresponding to the former [the latter] of these partitions by $\beta$ [respectively $\delta$ ]. Obviously, $\beta \subseteq \gamma$.

Now we aim to check that $\gamma$ is a fully invariant congruence. By the definition of $\gamma$ the condition $W_{1} \gamma W_{2}$ for distinct elements $W_{1}, W_{2} \in \mathrm{F}(\mathcal{X})$ implies either $W_{1}, W_{2} \in \mathfrak{W}$ or $\left\{W_{1}, W_{2}\right\}=\{\xi(U), \xi(S)\}$ or $\left\{W_{1}, W_{2}\right\}=\{\xi(V), \xi(T)\}$ for some automorphism $\xi$. We see that $\ell\left(W_{1}\right), \ell\left(W_{2}\right) \geq \ell$ always. Therefore if $W \in \mathrm{F}(\mathcal{X})$ then $\ell\left(W_{1} W\right), \ell\left(W_{2} W\right), \ell\left(W W_{1}\right), \ell\left(W W_{2}\right)>\ell$, whence $W_{1} W, W_{2} W, W W_{1}, W W_{2} \in \mathfrak{W}$. We see that $W_{1} W \gamma W_{2} W$ and $W W_{1} \gamma W W_{2}$ for any element $W$. This means that the relation $\gamma$ is a congruence. Now we shall prove that $\gamma$ is invariant under an arbitrary endomorphism $\varphi$ on $\mathrm{F}(\mathcal{X})$. Obviously, for any $W \in \mathrm{F}(\mathcal{X})$ we have $\ell(\varphi(W)) \geq \ell(W)$ and if $\ell(\varphi(W))=\ell(W)$ (i. e., if $\varphi$ maps every letter of $c(W)$ to a letter) then $|c(\varphi(W))| \leq|c(W)|$. This implies that $\varphi(\mathfrak{W}) \subseteq \mathfrak{W}$. It remains to prove that $\varphi\left(W_{1}\right) \gamma \varphi\left(W_{2}\right)$ for any distinct elements $W_{1}, W_{2} \in \mathrm{F}(\mathcal{X})$ with $W_{1} \gamma W_{2}$ and $W_{1}, W_{2} \notin \mathfrak{W}$. The latest means that either $\left\{W_{1}, W_{2}\right\}=\{\xi(U), \xi(S)\}$ or $\left\{W_{1}, W_{2}\right\}=\{\xi(V), \xi(T)\}$ for some $\xi$. We may suppose without loss of generality that $W_{1} \equiv \xi(U)$ and $W_{2} \equiv \xi(S)$. The following three cases are possible:

1) $\varphi$ maps some letter of $c(\xi(U))$ to an element of length $>1$;

2) $\varphi$ maps every letter of $c(\xi(U))$ to a letter and maps some two distinct letters of $c(\xi(U))$ to the same letter;

3) $\varphi$ maps all letters of $c(\xi(U))$ to distinct letters.

In the case 1) we have $\ell\left(\varphi\left(W_{1}\right)\right), \ell\left(\varphi\left(W_{2}\right)\right)>\ell$, while in the case 2) we have $\ell\left(\varphi\left(W_{1}\right)\right)=\ell\left(\varphi\left(W_{2}\right)\right)=\ell$ and $\left|c\left(\varphi\left(W_{1}\right)\right)\right|=\left|c\left(\varphi\left(W_{2}\right)\right)\right|<|c|$. In both the cases $\varphi\left(W_{1}\right), \varphi\left(W_{2}\right) \in \mathfrak{W}$. In the case 3$)$ there is an automorphism $\bar{\varphi}$ on $\mathrm{F}(\mathcal{X})$ such that $\bar{\varphi}\left(W_{1}\right) \equiv \varphi\left(W_{1}\right)$ and $\bar{\varphi}\left(W_{2}\right) \equiv \varphi\left(W_{2}\right)$, so the pair $\left\{\varphi\left(W_{1}\right), \varphi\left(W_{2}\right)\right\}=\{\bar{\varphi}(\xi(U)), \bar{\varphi}(\xi(S))\}$ is a $\gamma$-class. In all three cases 
$\varphi\left(W_{1}\right) \gamma \varphi\left(W_{2}\right)$. We have verified that $\gamma$ is a fully invariant congruence. Analogous arguments show that $\beta$ and $\delta$ are fully invariant congruences too.

Suppose that the variety $\mathcal{V}$ is a modular element of the lattice $\mathrm{L}(\mathcal{X})$. Then $\alpha$ is a modular element of the lattice $\mathrm{L}(\mathrm{F}(\mathcal{X}))$. Since $\beta \subseteq \gamma$, we have $(\alpha \vee \beta) \wedge \gamma=(\alpha \wedge \gamma) \vee \beta$. Further, $U \alpha V \beta T \alpha S$ and $U \gamma S$, whence $(U, S) \in(\alpha \vee \beta) \wedge \gamma=(\alpha \wedge \gamma) \vee \beta$. On the other hand, $U \notin \mathfrak{W}$ and $U \notin\{\xi(V), \xi(T)\}$ for any $\xi \in \operatorname{Aut}(\mathrm{F}(\mathcal{X}))$, so the set $\{U\}$ is a $\beta$-class. If $\{U\}$ is an $(\alpha \wedge \gamma)$-class then $\{U\}$ is an $((\alpha \wedge \gamma) \vee \beta)$-class too. But this fails because $(U, S) \in(\alpha \wedge \gamma) \vee \beta$. Thus there is an element $R$ such that $R \not \equiv U$ and $(U, R) \in \alpha \wedge \gamma$. In particular $U \gamma R$. By the definition of $\gamma$ the set $\{U, S\}$ is a $\gamma$-class. Hence $R \equiv S$. We see that $(U, S) \in \alpha \wedge \gamma$. In particular, $U \alpha S$, and we are done.

We have verified the 'modular half' of our lemma. Suppose now that the variety $\mathcal{V}$ is a lower-modular element of the lattice $\mathrm{L}(\mathcal{X})$. Then $\alpha$ is an upper-modular element of the lattice $\mathrm{L}(\mathrm{F}(\mathcal{X}))$. Put $\rho=\delta \wedge \alpha$. Since $\rho \subseteq \alpha$, we have $(\gamma \vee \rho) \wedge \alpha=(\gamma \wedge \alpha) \vee \rho$. Since $U \delta V$ and $U \alpha V$, we have $U \rho V$. Thus $S \gamma U \rho V \gamma T$ and $S \alpha T$, whence $(S, T) \in(\gamma \vee \rho) \wedge \alpha=(\gamma \wedge \alpha) \vee \rho$. On the other hand, $S \notin \mathfrak{W}$ and $S \notin\{\xi(U), \xi(V)\}$ for any automorphism $\xi$, so the set $\{S\}$ is a $\delta$-class. Hence $\{S\}$ is a $\rho$-class because $\rho \subseteq \delta$. If $\{S\}$ is a $(\gamma \wedge \alpha)$-class then $\{S\}$ is a $((\gamma \wedge \alpha) \vee \rho)$-class too. But this fails because $(S, T) \in(\gamma \wedge \alpha) \vee \rho$. Thus there is an element $Q$ such that $Q \not \equiv S$ and $(S, Q) \in \gamma \wedge \alpha$. In particular $S \gamma Q$. By the definition of $\gamma$ the set $\{S, U\}$ is a $\gamma$-class. Hence $Q \equiv U$. We see that $(S, U) \in \gamma \wedge \alpha$. In particular, $S \alpha U$, and we are done.

A semigroup variety $\mathcal{V}$ is called proper if $\mathcal{V} \neq \mathcal{S} \mathcal{E} \mathcal{M}$. For any word $w \equiv$ $x_{j_{1}} x_{j_{2}} \cdots x_{j_{n}}$ where $x_{j_{1}}, x_{j_{2}}, \ldots, x_{j_{n}}$ are (not necessarily dufferent) letters and for $1 \leq i \leq n$ we put $w[i] \equiv x_{j_{i}}$.

Corollary 3.2. If $\mathcal{V}$ is either a modular or a lower-modular proper semigroup variety then $\mathcal{V}$ is periodic.

Proof. Since the variety $\mathcal{V}$ is proper, it satisfies some non-trivial identity $w_{1}=w_{2}$. Suppose that $\mathcal{V}$ is not periodic. Then $\mathcal{V}$ is overcommutative by Lemma 2.9 , whence the identity $w_{1}=w_{2}$ is balanced by Lemma 2.10 . There is some $i \in\left\{1,2, \ldots, \ell\left(w_{1}\right)\right\}$ with $w_{1}[i] \not \equiv w_{2}[i]$. Put $x \equiv w_{1}[i]$ and $y \equiv w_{2}[i]$. Consider the words $u \equiv x^{2} w_{1}, v \equiv x^{2} w_{2}, s \equiv x y w_{1}$, and $t \equiv x y w_{2}$. The identities $u=v$ and $s=t$ are satisfied in $\mathcal{V}$ because they follow from $w_{1}=w_{2}$. The words $u, v, s$, and $t$ have the same length and the same content because $\ell\left(w_{1}\right)=\ell\left(w_{2}\right)$ and $c\left(w_{1}\right)=c\left(w_{2}\right)$. Now we aim to prove that these words are pairwise non-equivalent. Suppose that $u$ and $v$ are equivalent, so $v \equiv \xi(u)$ for some $\xi \in \operatorname{Aut}(\mathrm{F})$. Then $x^{2} w_{2} \equiv(\xi(x))^{2} \xi\left(w_{1}\right)$, whence $\xi(x) \equiv x$ and $\xi\left(w_{1}\right) \equiv w_{2}$. But $\xi\left(w_{1}\right) \equiv w_{2}$ implies $\xi(x) \equiv \xi\left(w_{1}[i]\right) \equiv w_{2}[i] \equiv y \not \equiv x$. The contradiction shows that $u$ and $v$ are non-equivalent. The words $s$ and $t$ are non-equivalent by analogous arguments. Finally, each of the words $u$ and $v$ is not equivalent to each of the words $s$ and $t$ because any of the equalities 
$u \equiv \xi(s), v \equiv \xi(s), u \equiv \xi(t)$, and $v \equiv \xi(t)$ for any $\xi \in \operatorname{Aut}(\mathrm{F})$ would imply $\xi(x y) \equiv x^{2}$ that is impossible. Since all words (in particular, the words $u, v$, $s$, and $t$ ) are $\mathcal{S E M}$-unstable, we can apply Lemma 3.1 with $\mathcal{X}=\mathcal{S E M}$ and conclude that $\mathcal{V}$ satisfies the identity $u=s$. This identity is not balanced because $\ell_{x}(u)=\ell_{x}\left(w_{1}\right)+2$, while $\ell_{x}(s)=\ell_{x}\left(w_{1}\right)+1$. Lemmas 2.9 and 2.10 imply that $\mathcal{V}$ is periodic.

Note that the fact that a proper lower-modular semigroup variety is periodic was verified earlier by another way in [13], Theorem 1.

Proposition 3.3. Let $\mathcal{X}$ be an overcommutative semigroup variety and $\mathcal{V}$ a periodic subvariety of $\mathcal{X}$. If $\mathcal{V}$ is either a modular or a lower-modular element of the lattice $\mathrm{L}(\mathcal{X})$ then $\mathcal{V}=\mathcal{M} \vee \mathcal{N}$ where $\mathcal{M}$ is one of the varieties $\mathcal{T}$ or $\mathcal{S} \mathcal{L}$, while $\mathcal{N}$ is a nil-variety.

Proof. At first, we shall prove that $\mathcal{V}=\mathcal{M} \vee \mathcal{N}$ where $\mathcal{M}$ is generated by a commutative monoid, while $\mathcal{N}$ is a nil-variety. Being periodic, the variety $\mathcal{V}$ satisfies the identity $x^{n}=x^{n+m}$ for some natural $n$ and $m$. We may assume without loss of generality that $n>1$. Put

$$
\begin{array}{ll}
u \equiv x y^{n+4 m+3} z^{n} q, & v \equiv x y^{n+2 m+3} z^{n+2 m} q, \\
s \equiv q y^{n+4 m+2} z^{n+1} x, & t \equiv q y^{n+2 m+2} z^{n+2 m+1} x .
\end{array}
$$

The variety $\mathcal{V}$ satisfies the identities $u=v$ and $s=t$. Further considerations are naturally divided into two cases.

Case 1: the words $u, v, s$, and $t$ are $\mathcal{X}$-unstable. These words have the same length and the same content. Besides that, these words are pairwise $\mathcal{X}$-non-equivalent because if $w_{1}, w_{2} \in\{u, v, s, t\}$ and $w_{1} \not \equiv w_{2}$ then $\max _{a \in c\left(w_{1}\right)}\left\{\ell_{a}\left(w_{1}\right)\right\} \neq \max _{a \in c\left(w_{2}\right)}\left\{\ell_{a}\left(w_{2}\right)\right\}$. Thus, we can apply Lemma 3.1 and conclude that the identity $u=s$, that is, the identity

$$
x y^{n+4 m+3} z^{n} q=q y^{n+4 m+2} z^{n+1} x
$$

holds in $\mathcal{V}$. Lemma 2.5 and its dual imply that this identity fails in the varieties $\mathcal{L} \mathcal{Z}, \mathcal{R Z}, \mathcal{P}$, and $\overleftarrow{\mathcal{P}}$. Now Lemma 2.6 applies and we conclude that $\mathcal{V}=\mathcal{M} \vee \mathcal{N}$ where $\mathcal{M}$ is generated by a monoid, while $\mathcal{N}$ is a nil-variety. Substituting 1 for $y$ and $z$ in the identity (1), we have that the identity $x q=q x$ holds in $\mathcal{M}$, so $\mathcal{M}$ is generated by a commutative monoid.

Case 2: at least one of the words $u, v, s$, and $t$ is $\mathcal{X}$-stable. Suppose that this word is $u$. Denote its $\mathcal{X}$-image by $U$. There is a non-trivial automorphism $\xi$ on $\mathrm{F}_{\{x, y, z, q\}}(\mathcal{X})$ with $\xi(U) \equiv U$. This automorphism performs some permutation on the set $\{x, y, z, q\}$. Since $\ell_{y}(U)>\ell_{z}(U)>\ell_{x}(U)=\ell_{q}(U)$, we have $\xi(y) \equiv y$ and $\xi(z) \equiv z$. Since the automorphism $\xi$ is non-trivial, we have $\xi(x) \equiv q$ and $\xi(q) \equiv x$. Thus the $\mathcal{X}$-images of the words $x y^{n+4 m+3} z^{n} q$ and $q y^{n+4 m+3} z^{n} x$ coincide. This means that the identity

$$
x y^{n+4 m+3} z^{n} q=q y^{n+4 m+3} z^{n} x
$$


holds in $\mathcal{X}$ and therefore, in $\mathcal{V}$. Lemma 2.5 and its dual imply that this identity fails in the varieties $\mathcal{L} \mathcal{Z}, \mathcal{R Z}, \mathcal{P}$, and $\overleftarrow{\mathcal{P}}$. Hence we may apply Lemma 2.6 and conclude that $\mathcal{V}=\mathcal{M} \vee \mathcal{N}$ where $\mathcal{M}$ is generated by a monoid, while $\mathcal{N}$ is a nil-variety. Substituting 1 for $y$ and $z$ in the identity (2), we have that the identity $x q=q x$ holds in $\mathcal{M}$, so $\mathcal{M}$ is generated by a commutative monoid. Analogous arguments may be used if one of the words $v, s$ or $t$ is $\mathcal{X}$-stable.

It remains to verify that $\mathcal{M}$ is one of the varieties $\mathcal{T}$ or $\mathcal{S} \mathcal{L}$. To do this, we note that the variety $\mathcal{V}$ satisfies the identities $u^{\prime}=v^{\prime}$ and $s^{\prime}=t^{\prime}$ where

$$
\begin{aligned}
& u^{\prime} \equiv x^{n+2 m+4} y^{n+2} z, \quad v^{\prime} \equiv x^{n+m+4} y^{n+m+2} z, \\
& s^{\prime} \equiv x^{n+2 m+3} y^{n+2} z^{2}, \quad t^{\prime} \equiv x^{n+m+3} y^{n+m+2} z^{2} .
\end{aligned}
$$

These words have the same length and the same content. Besides that, these words are pairwise $\mathcal{X}$-non-equivalent because if $w_{1}, w_{2} \in\left\{u^{\prime}, v^{\prime}, s^{\prime}, t^{\prime}\right\}$ and $w_{1} \not \equiv w_{2}$ then either $\max _{a \in c\left(w_{1}\right)}\left\{\ell_{a}\left(w_{1}\right)\right\} \neq \max _{a \in c\left(w_{2}\right)}\left\{\ell_{a}\left(w_{2}\right)\right\}$ or $\min _{a \in c\left(w_{1}\right)}\left\{\ell_{a}\left(w_{1}\right)\right\} \neq$ $\min _{a \in c\left(w_{2}\right)}\left\{\ell_{a}\left(w_{2}\right)\right\}$. Finally, the words $u^{\prime}, v^{\prime}, s^{\prime}$, and $t^{\prime}$ are $\mathcal{X}$-unstable because $\ell_{x}(w)>\ell_{y}(w)>\ell_{z}(w)$ for any $w \in\left\{u^{\prime}, v^{\prime}, s^{\prime}, t^{\prime}\right\}$. By Lemma 3.1 the variety $\mathcal{V}$ satisfies the identity $u^{\prime}=s^{\prime}$, that is, the identity

$$
x^{n+2 m+4} y^{n+2} z=x^{n+2 m+3} y^{n+2} z^{2} .
$$

Substituting 1 for $x$ and $y$ in this identity, we have that the identity $z=z^{2}$ holds in $\mathcal{M}$. Thus $\mathcal{M} \subseteq \mathcal{S} \mathcal{L}$, whence $\mathcal{M}$ is one of the varieties $\mathcal{T}$ or $\mathcal{S} \mathcal{L}$.

Now we are well prepared to complete the proofs of Theorems 1.4 and 1.6 and to give new proofs of Propositions 1.1 and 1.3.

Proposition 1.1 [Theorem 1.4] is directly implied by Proposition 3.3 with $\mathcal{X}=\mathcal{S E M}$ [respectively $\mathcal{X}=\mathcal{C O} \mathcal{M}$ ] and Corollary 3.2 [Lemma 2.9].

Proof of Proposition 1.3 and Theorem 1.6. In both the statements, sufficiency immediately follows from Lemmas 2.3 and 2.1. One can prove necessity. Let $\mathcal{V}$ be a [commutative] lower-modular [in Com] semigroup variety with $\mathcal{V} \neq \mathcal{S E M}$ [respectively $\mathcal{V} \neq \mathcal{C O} \mathcal{M}]$. Then the variety $\mathcal{V}$ is periodic by Corollary 3.2 [Lemma 2.9]. Applying Proposition 3.3 with $\mathcal{X}=\mathcal{S E M}$ [respectively $\mathcal{X}=\mathcal{C O} \mathcal{M}$ ], we conclude that $\mathcal{V}=\mathcal{M} \vee \mathcal{N}$ where $\mathcal{M}$ is one of the varieties $\mathcal{T}$ or $\mathcal{S} \mathcal{L}$, while $\mathcal{N}$ is a nil-variety. Lemma 2.1 implies that the variety $\mathcal{N}$ is lower-modular [in $\mathbf{C o m}$ ]. Then the variety $\mathcal{N}$ is 0 -reduced [in Com] by Lemma 2.3.

To prove Theorem 1.5, we need some additional auxiliary results.

Lemma 3.4. An identity $v=0$ follows from an identity system $\{x y=$ $y x, u=0\}$ if and only if there is some (possibly empty) word $w$ and some endomorphism $\xi$ on $\mathrm{F}$ such that the identity $v=w \xi(u)$ is balanced.

Proof. Let $U$ be the $\mathcal{C O M}$-image of the word $u$. Consider the set

$$
\mathfrak{W}=\{W \zeta(U) \mid W \in \mathrm{F}(\mathcal{C O} \mathcal{M}), \zeta \in \operatorname{End}(\mathrm{F}(\mathcal{C O} \mathcal{M}))\} .
$$


It is evident that $\mathfrak{W}$ is an ideal of the semigroup $\mathrm{F}(\mathcal{C O M})$ and that $\zeta(\mathfrak{W}) \subseteq$ $\mathfrak{W}$ for any $\zeta \in \operatorname{End}(\mathrm{F}(\mathcal{C O} \mathcal{M}))$. Hence the relation $\alpha$ on $\mathrm{F}(\mathcal{C O} \mathcal{M})$ defined by the rule

$$
W_{1} \alpha W_{2} \text { if and only if either } W_{1} \equiv W_{2} \text { or } W_{1}, W_{2} \in \mathfrak{W}
$$

is a fully invariant congruence on $\mathrm{F}(\mathcal{C O} \mathcal{M})$. Put $\mathcal{V}=\operatorname{var}\{x y=y x, u=0\}$ and consider the fully invariant congruence $\alpha^{\prime}$ corresponding to $\mathcal{V}$. We shall prove that $\alpha=\alpha^{\prime}$. Since $\alpha^{\prime}$ corresponds to a 0-reduced in Com variety, it has only one non-singleton class $\mathfrak{W}^{\prime}$. The set $\mathfrak{W}^{\prime}$ is an ideal of the semigroup $\mathrm{F}(\mathcal{C O M})$. This ideal contains $U$ and satisfies $\zeta\left(\mathfrak{W}^{\prime}\right) \subseteq \mathfrak{W}^{\prime}$ for any $\zeta \in \operatorname{End}(\mathrm{F}(\mathcal{C O} \mathcal{M}))$ because the congruence $\alpha^{\prime}$ is fully invariant. Therefore $\zeta(U) \in \mathfrak{W}^{\prime}$ for any endomorphism $\zeta$. Hence $W \zeta(U) \in \mathfrak{W}^{\prime}$ for any $W \in \mathrm{F}(\mathcal{C O M})$ because $\mathfrak{W}^{\prime}$ is an ideal. We see that $\mathfrak{W} \subseteq \mathfrak{W}^{\prime}$, whence $\alpha \subseteq \alpha^{\prime}$. Further, $\mathfrak{W}$ is a zero of the factor semigroup $\mathrm{F}(\mathcal{C O M}) / \alpha$. Since the inclusion $\zeta(U) \in \mathfrak{W}$ holds for any endomorphism $\zeta$ on $\mathrm{F}(\mathcal{C O M})$, the identity $u=0$ holds in $\mathrm{F}(\mathcal{C O M}) / \alpha$. Hence $\mathrm{F}(\mathcal{C O} \mathcal{M}) / \alpha \in \mathcal{V}$, so $\alpha^{\prime} \subseteq \alpha$. We have proved that $\alpha=\alpha^{\prime}$ and hence $\mathfrak{W}=\mathfrak{W}^{\prime}$.

An identity $v=0$ follows from the system $\{x y=y x, u=0\}$ if and only if it holds in $\mathcal{V}$. This is so if and only if the $\mathcal{C O} \mathcal{M}$-image $V$ of the word $v$ belongs to $\mathfrak{W}^{\prime}=\mathfrak{W}$, i. e. $V \equiv W \zeta(U)$ for some $W \in \mathrm{F}(\mathcal{C O M})$ and some $\zeta \in \operatorname{End}(\mathrm{F}(\mathcal{C O} \mathcal{M}))$. But every element of $\mathrm{F}(\mathcal{C O} \mathcal{M})$ is a $\mathcal{C O} \mathcal{M}$-image of some word and every endomorphism on $\mathrm{F}(\mathcal{C O} \mathcal{M})$ has the form $\varphi \xi$ where $\xi$ is an endomorphism on $\mathrm{F}$, while $\varphi$ is the natural homomorphism from $\mathrm{F}$ to $\mathrm{F}(\mathcal{C O M})$. Therefore the equality $V \equiv W \zeta(U)$ is the equality of $\mathcal{C O} \mathcal{M}$ images of words $v$ and $w \xi(u)$ for some word $w$ and some $\xi \in \operatorname{End}(\mathrm{F})$. So this equality means that the identity $v=w \zeta(u)$ holds in $\mathcal{C O M}$, whence it is balanced by Lemma 2.10 .

Corollary 3.5. Words $u$ and $v$ are $\mathcal{C O} \mathcal{M}$-equivalent if and only if the identity systems $\{x y=y x, u=0\}$ and $\{x y=y x, v=0\}$ are equivalent.

Proof. Necessity. If $u$ and $v$ are $\mathcal{C O} \mathcal{M}$-equivalent then there is some automorphism $\xi$ on $\mathrm{F}$ such that the identity $v=\xi(u)$ holds in $\mathcal{C O} \mathcal{M}$, whence it is balanced by Lemma 2.10. Then the identity $v=0$ follows from the system $\{x y=y x, u=0\}$ by Lemma 3.4. By symmetry, the identity $u=0$ follows from the system $\{x y=y x, v=0\}$. Hence these two systems are equivalent.

Sufficiency. Suppose that the systems $\{x y=y x, u=0\}$ and $\{x y=$ $y x, v=0\}$ are equivalent. By Lemma 3.4 there are some (possibly empty) words $a$ and $b$ and some automorphisms $\xi$ and $\zeta$ on F such that the identities $u=a \xi(v)$ and $v=b \zeta(u)$ are balanced. Then the identity $u=a \xi(b \zeta(u))$ is balanced too. Hence $\ell(u)=\ell(a \xi(b \zeta(u)))$. But this is possible only if the words $a$ and $b$ are empty and the endomorphism $\xi \zeta$ maps every letter of $u$ to a letter. Further, $c(u)=c(\xi \zeta(u))$ because the identity $u=\xi \zeta(u)$ is balanced. This means that $\xi \zeta$ maps distinct letters of $u$ to distinct letters. Therefore both endomorphisms $\xi$ and $\zeta$ have the same property and the restrictions of $\xi$ and $\zeta$ on the semigroups $\mathrm{F}_{c(v)}$ and $F_{c(u)}$ respectively are 
isomorphisms between these semigroups. Thus the identity $u=\xi(v)$ is balanced and $\xi$ perfoms an isomorphism from $\mathrm{F}_{c(v)}$ to $\mathrm{F}_{c(u)}$. Therefore $u$ is $\mathcal{C O} \mathcal{M}$-equivalent to $v$.

Proof of Theorem 1.5. Suppose that a commutative nil-variety $\mathcal{V}$ is modular in Com and consider any identity $u=v$ satisfied in $\mathcal{V}$. Suppose that the identities $u=0$ and $v=0$ are not satisfied in $\mathcal{V}$. We aim to prove that the identity $u=v$ follows from the commutative law and some substitutive identity satisfied in $\mathcal{V}$.

By Lemma 2.7 there is an Abelian periodic group variety $\mathcal{G}$ such that

$$
\mathcal{G} \vee \mathcal{V}=\mathcal{G} \vee \mathrm{ZR}(\mathcal{V})
$$

Put $\mathcal{X}=\operatorname{var}\{x y=y x, u=0\}, \mathcal{Y}=\operatorname{var}\{x y=y x, v=0\}$, and $\mathcal{Z}=\mathcal{X} \vee \mathcal{G}$. The variety $\mathcal{G}$ satisfies the identity $x^{n} y=y$ for some $n$ and therefore the identity $x^{n} u=u$. This identity holds also in $\mathcal{X}$, so it holds in $\mathcal{Z}$ and therefore in $\mathcal{V} \wedge \mathcal{Z}$. Being a nil-variety, $\mathcal{V} \wedge \mathcal{Z}$ satisfies the identity $u=0$ by Lemma 2.8(ii). The identity $u=v$ holds in $\mathcal{V}$, whence $u=v=0$ holds in $\mathcal{V} \wedge \mathcal{Z}$, i. e.

$$
\mathcal{V} \wedge \mathcal{Z} \subseteq \mathcal{Y}
$$

So we have

$$
\begin{array}{rlrl}
(\mathrm{ZR}(\mathcal{V}) \wedge \mathcal{X}) \vee \mathcal{G} & \subseteq(\mathrm{ZR}(\mathcal{V}) \vee \mathcal{G}) \wedge(\mathcal{X} \vee \mathcal{G}) & & \\
& =(\mathrm{ZR}(\mathcal{V}) \vee \mathcal{G}) \wedge \mathcal{Z} & & \\
& =(\mathcal{V} \vee \mathcal{G}) \wedge \mathcal{Z} & & \text { by }(3) \\
& =(\mathcal{V} \wedge \mathcal{Z}) \vee \mathcal{G} & & \text { because } \mathcal{V} \text { is modular } \\
& \subseteq \mathcal{Y} \vee \mathcal{G} & & \text { in Com and } \mathcal{G} \subseteq \mathcal{Z} \\
& & \text { by }(4) .
\end{array}
$$

Thus $(\mathrm{ZR}(\mathcal{V}) \wedge \mathcal{X}) \vee \mathcal{G} \subseteq \mathcal{Y} \vee \mathcal{G}$. The identity $v=v x^{n}$ holds in $\mathcal{Y} \vee \mathcal{G}$, so it holds in $(\operatorname{ZR}(\mathcal{V}) \wedge \mathcal{X}) \vee \mathcal{G}$ and therefore in $\operatorname{ZR}(\mathcal{V}) \wedge \mathcal{X}$. Since $\operatorname{ZR}(\mathcal{V}) \wedge \mathcal{X}$ is a nil-variety, it satisfies the identity $v=0$ by Lemma 2.8(ii). Hence there is a deduction of this identity from identities of the varieties $\operatorname{ZR}(\mathcal{V})$ and $\mathcal{X}$, that is, a sequence of words $w_{0}, w_{1}, \ldots, w_{n}$ such that $v \equiv w_{0}$ and each of the identities $w_{0}=w_{1}, w_{1}=w_{2}, w_{n-1}=w_{n}$, and $w_{n}=0$ holds in one of the varieties $\operatorname{ZR}(\mathcal{V})$ or $\mathcal{X}$. We may assume without loss of generality that $w_{0}, w_{1}, \ldots, w_{n}$ is the shortest sequence with these properties. In particular, for any $i=0,1, \ldots, n-1$, none of the varieties $\operatorname{ZR}(\mathcal{V})$ and $\mathcal{X}$ satisfies the identity $w_{i}=0$, and the identity $w_{i}=w_{i+1}$ does not hold in both the varieties $\operatorname{ZR}(\mathcal{V})$ and $\mathcal{X}$ simultaneously. Suppose that $n>0$. Let $i \in$ $\{0,1, \ldots, n-1\}$. Since the varieties $\operatorname{ZR}(\mathcal{V})$ and $\mathcal{X}$ are 0 -reduced in Com and none of these varieties satisfies the identity $w_{i}=0$, the identity $w_{i}=$ $w_{i+1}$ holds in $\mathcal{C O} \mathcal{M}$ and therefore, in both the varieties $\mathrm{ZR}(\mathcal{V})$ and $\mathcal{X}$. A contradiction shows that $n=0$. Thus the identity $v=0$ holds in one of the varieties $\operatorname{ZR}(\mathcal{V})$ or $\mathcal{X}$. But this identity fails in $\operatorname{ZR}(\mathcal{V})$ because it fails in $\mathcal{V}$. So $v=0$ holds in $\mathcal{X}$. Analogously, considering the variety $\mathcal{Y} \vee \mathcal{G}$ rather than 
$\mathcal{X} \vee \mathcal{G}$, we can prove that the identity $u=0$ holds in $\mathcal{Y}$. This means that $\mathcal{X}=\mathcal{Y}$, so the identity systems $\{x y=y x, u=0\}$ and $\{x y=y x, v=0\}$ are equivalent. By Lemma 3.5 the words $u$ and $v$ are $\mathcal{C O} \mathcal{M}$-equivalent. Hence there is some automorphism $\xi$ on $\mathrm{F}(\mathcal{C O} \mathcal{M})$ such that the identity $u=\xi(v)$ holds in $\mathcal{C O} \mathcal{M}$. In particular, this identity is balanced (by Lemma 2.10) and holds in $\mathcal{V}$. The identity $v=\xi(v)$ holds in $\mathcal{V}$ because the identities $u=v$ and $u=\xi(v)$ hold in $\mathcal{V}$. If $c(v) \neq c(\xi(v))$ then $\mathcal{V}$ satisfies the identity $v=0$ by Lemma 2.8(i). But this is not true. Therefore $c(v)=c(\xi(v))$. This means that the identity $v=\xi(v)$ is substitutive. Being balanced, the identity $u=\xi(v)$ follows from the commutative law. The identities $u=\xi(v)$ and $v=\xi(v)$ imply $u=v$. Therefore, $u=v$ follows from $v=\xi(v)$ and the commutative law. Since the identity $v=\xi(v)$ is substitutive and holds in $\mathcal{V}$, we are done.

Theorem 1.5 and the 'commutative half' of Lemma 2.2 provide a necessary and a sufficient condition for a commutative nil-variety to be modular in Com respectively. The gap between these conditions seems to be not very large. But the necessary condition is not a sufficient one, while the sufficient condition is not a necessary one. Indeed, it may be checked that the variety $\operatorname{var}\left\{x y z t=x^{3}=0, x^{2} y=y^{2} x, x y=y x\right\}$ is modular in Com although it is not 0 -reduced in Com, while the variety $\operatorname{var}\left\{x^{5}=0, x^{3} y^{2}=y^{3} x^{2}, x y=y x\right\}$ is not modular in Com although it is given within $\mathcal{C O M}$ by 0 -reduced and substitutive identities only.

\section{Corollaries}

It was verified in [10] that a lower-modular semigroup variety is modular. Theorem 1.6, together with results of [9], implies the following 'commutative analog' of this fact.

Corollary 4.1. Every lower-modular in Com variety is modular in Com.

Proof. Let $\mathcal{V}$ be a lower-modular in Com variety. We may assume that $\mathcal{V} \neq \mathcal{C O} \mathcal{M}$. Then Theorem 1.6 implies that $\mathcal{V}=\mathcal{M} \vee \mathcal{N}$ where $\mathcal{M}$ is one of the varieties $\mathcal{T}$ or $\mathcal{S} \mathcal{L}$, while $\mathcal{N}$ is a 0 -reduced in Com variety. The variety $\mathcal{N}$ is modular in Com by Lemma 2.2. It remains to refer to Lemma 2.1.

By the way, we note that all five other possible implications between properties of being a modular in Com variety, a lower-modular in Com variety and an upper-modular in Com variety are fail. For instance:

- the variety $\operatorname{var}\left\{x y z t=x^{3}=0, x^{2} y=y^{2} x, x y=y x\right\}$ is modular in Com (as we have already mentioned at the end of Section 3) but not lower-modular in Com (by Theorem 1.6);

- the variety var $\left\{x^{3}=0, x y=y x\right\}$ is modular in Com (by Lemma 2.2) and lower-modular in Com (by Lemma 2.3) but not upper-modular in Com (by Lemma 2.4);

- the variety of Abelian groups of a prime exponent is upper-modular in Com (because this variety is an atom of the lattice Com) but 
neither modular in Com (by Theorem 1.4) nor lower-modular in Com (by Theorem 1.6).

It was proved in [9], Theorem 1.2, that a commutative semigroup variety is neutral in Com if and only if it is both distributive and codistributive in Com. This assertion is generalized by the following

Corollary 4.2. For a commutative semigroup variety $\mathcal{V}$, the following are equivalent:

a) $\mathcal{V}$ is both lower-modular and upper-modular in Com;

b) $\mathcal{V}$ is neutral in $\mathbf{C o m}$;

c) either $\mathcal{V}=\mathcal{C O} \mathcal{M}$ or $\mathcal{V}=\mathcal{M} \vee \mathcal{N}$ where $\mathcal{M}$ is one of the varieties $\mathcal{T}$ or $\mathcal{S} \mathcal{L}$, while $\mathcal{N}$ satisfies the identities $x^{2} y=0$ and $x y=y x$.

Proof. The equivalence of the statements b) and c) is proved in [9], Theorem 1.2 , while the implication $\mathrm{b}) \longrightarrow$ a) is evident.

a) $\longrightarrow c)$. Suppose that a variety $\mathcal{V}$ is both lower-modular and uppermodular in Com. We may assume that $\mathcal{V} \neq \mathcal{C O M}$. Then Theorem 1.6 implies that $\mathcal{V}=\mathcal{M} \vee \mathcal{N}$ where $\mathcal{M}$ is one of the varieties $\mathcal{T}$ or $\mathcal{S} \mathcal{L}$, while $\mathcal{N}$ is a 0 -reduced in Com variety. The variety $\mathcal{N}$ is upper-modular in $\mathbf{C o m}$ by Lemma 2.1. It remains to refer to Lemma 2.4.

Note that the analog of Corollary 4.2 for the lattice SEM also is true ([14], Corollary 3.5).

\section{REFERENCES}

[1] S. Burris and E.Nelson, Embedding the dual of $\Pi_{m}$ in the lattice of equational classes of commutative semigroups, Proc. Amer. Math. Soc., 30 (1971), 37-39.

[2] E. A. Golubov and M. V. Sapir, Residually small varieties of semigroups, Izvestiya VUZ. Matematika, No. 11 (1982), 21-29, in Russian; Engl. translation: Soviet Math. Izv. VUZ, 26, No. 11 (1982), 25-36.

[3] G. Grätzer, General Lattice Theory, 2-nd ed., Birkhauser Verlag, Basel (1998).

[4] J. Ježek, The lattice of equational theories. Part I: modular elements, Czechosl. Math. J., 31 (1981), 127-152.

[5] J. Ježek and R. N. McKenzie, Definability in the lattice of equational theories of semigroups, Semigroup Forum, 46 (1993), 199-245.

[6] A. Kisielewicz, Varieties of commutative semigroups, Trans. Amer. Math. Soc., 342 (1994), 275-306.

[7] P. Perkins, Bases for equational theories of semigroups, J. Algebra, 11 (1969), 298-314.

[8] P. Pudlák and J. Túma, Every finite lattice can be embedded in a finite partition lattice, Algebra Universalis, 10 (1980), 74-95.

[9] V. Yu. Shaprynskiř, Distributive and neutral elements of the lattice of commutative semigroup varieties, Izvestiya VUZ. Matematika, accepted, in Russian.

[10] V. Yu. Shaprynskiř and B. M. Vernikov, Lower-modular elements of the lattice of semigroup varieties. III, Acta. Sci. Math. (Szeged), accepted.

[11] L. N. Shevrin, B. M. Vernikov, and M. V. Volkov, Lattices of semigroup varieties, Izvestiya VUZ. Matematika, No.3 (2009), 3-36, in Russian; Engl. translation: Russian Math. Izv. VUZ, 53, No. 3 (2009), 1-28.

[12] B. M. Vernikov, On modular elements of the lattice of semigroup varieties, Comment. Math. Univ. Carol., 48 (2007), 595-606. 
[13] B. M. Vernikov, Lower-modular elements of the lattice of semigroup varieties, Semigroup Forum, 75 (2007), 554-566.

[14] B. M. Vernikov, Lower-modular elements of the lattice of semigroup varieties. II, Acta Sci. Math. (Szeged), 74 (2008), 539-556.

[15] B. M. Vernikov and V. Yu. Shaprynskiř, Distributive elements of the lattice of semigroup varieties, Algebra i Logika, 49 (2010), 303-330, in Russian; Engl. translation: Algebra and Logic, 49 (2010), 201-220.

[16] B. M. Vernikov and M. V. Volkov, Lattices of nilpotent semigroup varieties, Algebraicheskie Systemy i Ih Mnogoobraziya (Algebraic Systems and Their Varieties), Sverdlovsk: Ural State University (1988), 53-65, in Russian.

[17] M. V. Volkov, Semigroup varieties with modular subvariety lattices, Izvestiya VUZ. Matematika, No. 6 (1989), 51-60, in Russian; Engl. translation: Soviet Math. Izv. VUZ, 33, No. 6 (1989), 48-58.

[18] M. V. Volkov, Modular elements of the lattice of semigroup varieties, Contrib. General Algebra, 16 (2005), 275-288.

Department of Mathematics and Mechanics, Ural State University, Lenina 51, 620083 EKATERINBURG, Russia

E-mail address: vshapr@yandex.ru 